\title{
"Who has to do it at the end of the day? Programme officials or hospital authorities?" Airborne infection control at drug resistant tuberculosis (DR-TB) centres of Karnataka, India: a mixed-methods study
}

Kibballi Madhukeshwar Akshaya ${ }^{1 *}$ (D), Hemant Deepak Shewade², Ottapura Prabhakaran Aslesh, Sharath Burugina Nagaraja ${ }^{4}$, Abhay Subashrao Nirgude ${ }^{1}$, Anil Singarajipura ${ }^{5}$ and Anil G. Jacob²

\begin{abstract}
Background: Drug resistant tuberculosis (DR-TB) centers admit patients with DR-TB for initiation of treatment and thereby concentrate the patients under one setting. It becomes imperative to assess the compliance of DR-TB centres to national airborne infection control (AIC) guidelines and explore the provider perspectives into reasons for unsatisfactory compliance.

Methods: This mixed methods study (triangulation design) was carried out across all the six DR-TB centers of Karnataka state, India, between November 2016 and April 2017. Non-participant observation using a structured format was carried out at the DR-TB wards $(n=6)$, outpatient departments $(n=6)$, patient waiting areas outside outpatient departments $(n=6)$ and culture and drug susceptibility testing laboratories $(n=3)$. Structured interviews of admitted patients $(n=30)$ were done to assess the knowledge on cough hygiene and sputum disposal. Key informant interviews (KIIs) of health care providers $(n=20)$ were done. Manual descriptive content analysis was done to analyse the transcripts of Klls.

Results: The findings related to compliance in non-participant observation were corroborated by Klls. All the laboratories were consistently implementing the AIC guidelines. Compliance to hand hygiene, wet mopping and ventilation measures were satisfactory in four or more DR-TB wards. The non-availability of N95 masks in wards as well as outpatient departments was staggering. Sputum disposal without prior disinfection and the lack of display materials on cough hygiene and patient education was common. Patient fast tracking in outpatient department waiting areas and visitor restrictions in wards were lacking. Trainings on AIC measures were uncommon. About half and one-third of patients admitted had satisfactory knowledge regarding sputum disposal and situations demanding mask respectively. The reasons for unsatisfactory compliance to AIC guidelines were poor coordination between programme and hospital authorities leading to lack of ownership; ineffective or non-existent infection control committees; vacant posts of medical officers; and attitudes of health care delivery staff.

Conclusion: Compliance with AIC guidelines in DR-TB centers of Karnataka was sub-optimal. The reasons identified require urgent attention of the programme managers and hospital authorities.
\end{abstract}

Keywords: Infection control, Drug-resistant tuberculosis, MDR tuberculosis, Hospital infections, SORT IT

\footnotetext{
* Correspondence: docakshay@gmail.com

1 Department of Community Medicine, Yenepoya Medical College, Yenepoya

University, Mangaluru 575018, India

Full list of author information is available at the end of the article
} 


\section{Background}

Despite Tuberculosis (TB) being a curable disease through the use of standardized drugs, the increasing resistance to these drugs in the form of multi-drug resistant tuberculosis (MDR-TB), defined as resistance to isoniazid and rifampicin or rifampicin only, is a growing public health problem across the globe. India has the highest burden of MDR-TB patients in the world. An estimated 130,000 MDR-TB patients occur annually in India which includes 79,000 estimated among notified pulmonary cases $[1,2]$. India follows programmatic management of drug resistant TB (PMDT) guidelines within the revised national TB control programme (RNTCP) [3]. These services have expanded across all the states and union territories of India covering the whole population by 2013 [2].

Under PMDT, patients with MDR-TB are treated primarily on domiciliary basis after a brief period of inpatient care during treatment initiation in Drug Resistant TB (DR-TB) centers (one for every 10 million population). These are located within the premises of a medical college hospital or a tertiary public health facility (catering to patients other than MDR-TB as well), under the auspices of departments of pulmonary medicine or internal medicine (if the former does not exist). Each DR-TB centre has a separate ward for male and female patients, PMDT services [culture and drug susceptibility testing (CDST) and ancillary drugs for management of adverse drug reactions] and availability of relevant specialties or linkages for these services established [3]. Currently there is a network of 143 DR-TB centres across the country which are supported by 54 linked DR-TB centres (decentralized clinical unit under a DR-TB centre which provides treatment services but reporting lies with the parent DR-TB centre) [2].

In India, the treatment approach for patients with DR-TB concentrates the drug resistant patients under one roof during the initiation of treatment and also during the management of complications. Patients with MDR-TB in these settings pose a potential source of infection to the health care providers and other patients in these facilities who may be immune-compromised [4-7]. The transmission of the infection can be reduced by implementing effective airborne infection control measures in these settings $[8,9]$.

The national airborne infection control (AIC) guidelines were drafted in 2010 which proposed standards for AIC across various health facilities including high risk settings like MDR-TB wards [10]. A study assessing the implementation of these guidelines which was carried out across 35 healthcare facilities (these included four MDR-TB wards) in three states of India revealed that AIC practices were poorly implemented [11]. After rapid expansion of the PMDT services in the country there is scarce information on implementation of these guidelines at DR-TB centres and the barriers faced by the health care providers in complying with these guidelines.
Hence, a study was conducted to assess the compliance of DR-TB centres to National AIC guidelines (2010) and explore the provider perspectives related to the barriers encountered for implementation in the state of Karnataka, India (2016-17).

\section{Methods \\ Study design}

It was a cross-sectional study involving a mixed-methods approach (triangulation design) where compliance to the implementation of AIC guidelines were assessed through non-participant observation of DR-TB centers, structured interviews of admitted patients and key informant interviews (KIIs) of health care providers [12].

\section{Study setting \\ General setting}

Karnataka is the eighth largest state of India in terms of both area and population and is situated on the western edge of the Deccan plateau. The state has an area of $191,791 \mathrm{sq}$. $\mathrm{km}$. and a population of 61.1 million [13]. It is divided into 30 administrative districts.

\section{DR-TB centres}

PMDT services in Karnataka are delivered through six functional DR-TB centres situated at Bengaluru, Mysuru, Mangaluru, Hubballi, Ballari and Kalaburgi (Fig. 1). Patients with $\mathrm{TB}$ detected with rifampicin resistance are registered for MDR-TB treatment. Patients undergo second-line DST of patients with MDR-TB fit the presumptive extensively drug resistant TB criteria. A total of 1338 patients were detected with MDR-TB and 1099 of them were registered and initiated on treatment in these centres in 2016 [2, 3].

DR-TB centers are located in tertiary public healthcare facilities known as TB and chest diseases hospitals (Bengaluru, Mysuru, Ballari) as well as government medical college/district hospitals (Hubballi, Kalaburgi, Mangaluru). These centers were set up with one-time provision of up to USD 15500 from RNTCP for up-gradation of the existing wards to incorporate airborne infection control measures. The DR-TB centers are administratively controlled by the health of the institution (director or medical superintendent - administrator of these tertiary public health facilities/ medical college/ district hospitals). The routine health care services are provided by a specialist working in pulmonary medicine or internal medicine department (nodal officer), nursing and housekeeping staff. The national TB control programme supports the DR-TB centers by providing drugs and additional human resources that include a medical officer, a counsellor and a statistical assistant. However, the routine clinical laboratory investigation facilities for pre treatment evaluation and monitoring, ancillary drugs as 


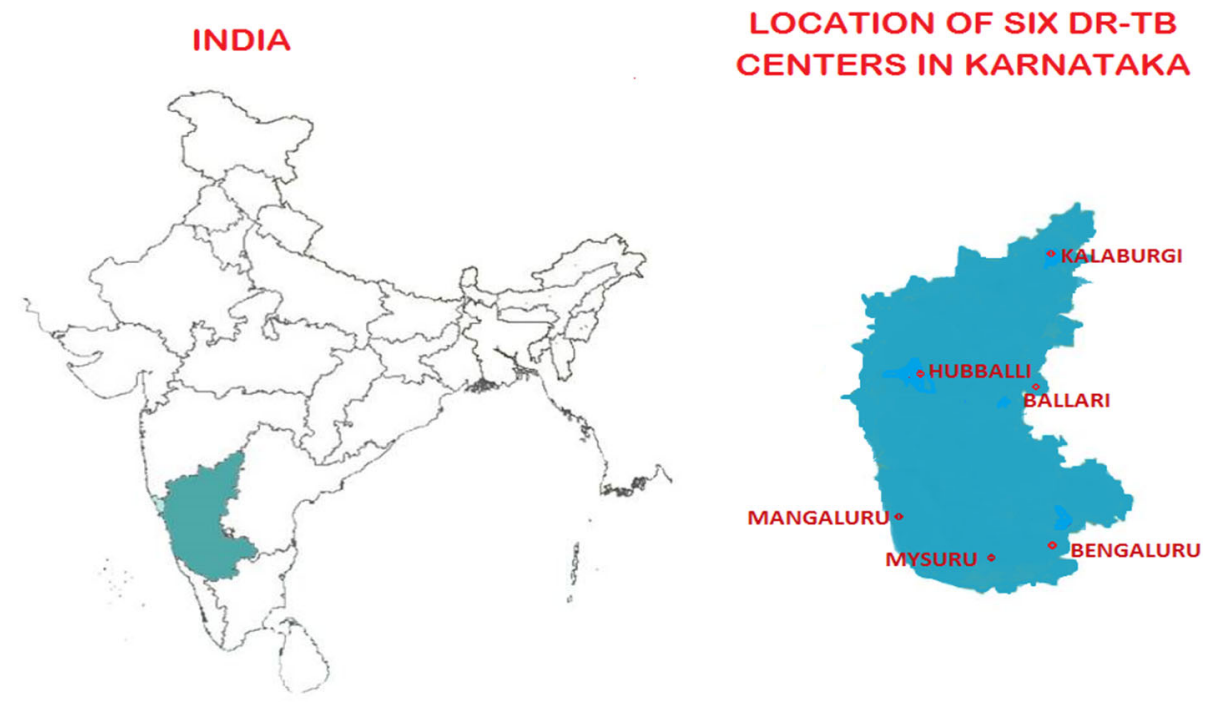

Fig. 1 The six drug resistant TB (DR-TB) centres in the state of Karnataka, India (2016-17)

per DR-TB centre committee's advice, drugs required for management of adverse drug reactions should be made available from the institute as part of their commitment for which no reimbursement will be available from the programme [3].

\section{Study population}

All the six DR-TB centers in Karnataka, India, were included in the study. These contained 21 units for AIC assessment: chest medicine/ internal medicine outpatient department $(\mathrm{OPD})(n=6)$, waiting areas outside the OPDs $(n=6)$, DR-TB wards $(n=6)$ and CDST laboratories $(n=3)$. All the in-patients admitted in the DR-TB wards at the time of visit by principal investigator (PI) drawn from the line list prepared by the staff nurse on duty were also interviewed.

Health care providers across four different cadres involved in providing DR-TB care at these facilities were interviewed $(n=20)$. We intended to interview all the administrators and medical officers of the DR$\mathrm{TB}$ center. In the absence of the medical officer, the nodal officer was interviewed subject to availability. The nursing and the housekeeping staff who were on duty at the time of visit of the PI were interviewed based on convenience sampling.

\section{Data collection, variables and sources of data}

Data collection was done between November 2016 and April 2017. A checklist (dichotomous variables) drawn from the national AIC guidelines [10] was administered through non-participant observation (Additional file 1: Annex S1) and in-patients with MDR-TB were interviewed about cough hygiene and sputum disposal using a structured closed ended interviewer administered questionnaire [translated into the local language (Kannada), back translated for content validation and pretested at Mangaluru]. (Additional file 1: Annex S2) Systematic qualitative enquiry was done through key informant interviews of health care providers which included administrators $(n=4)$, medical officers or nodal officers $(n=4)$, staff nurses $(n=6)$ and housekeeping employees $(n=6)$. Separate interview guides were prepared for these different cadres of health care staff and further modified based on the results of our nonparticipant observations and pre-testing at Mangaluru. (Additional file 1: Annex S3).

The interviewer, a 36 years old male (KMA), a faculty member (M.B.B.S; M.D.; D.N.B.) in a medical college in the region was trained in the use of qualitative research methods. The interviews were conducted in English or local language (Kannada) at their workplace after obtaining their written permission and consent to participate in the study. Administrators and medical officers or nodal officers were a priori informed over phone about the purpose of visit and their expected role in the interview. Only the participant and the researcher were present during the interview. Depending on participant comfort, consent was sought for audio recording; verbatim written notes were taken during the interview. The duration of the interviews varied between 5 and 20 min.

\section{Data entry and analysis}

Quantitative data collected were double-entered, validated and analysed using EpiData (version 3.1 for entry and version 2.2.2.183 for analysis, EpiData Association, Odense, Denmark). Frequency and proportions were used to summarise the key analytic outputs. 
The interviews were transcribed and translated (Kannada to English), if required, within $48 \mathrm{~h}$ based on the notes and audio records of the interviews (KMA). These transcripts were compiled and read to become familiar with the data. Manual descriptive content analysis was done to analyse the transcripts. Categories were decided a priori and codes were generated inductively [12, 14]. These were reviewed by the second investigator (HDS) to reduce bias, increase inter-coder reliability and enhance interpretative credibility. The decision on coding rules and theme generation was done by using standard procedures and based on consensus [15]. Differences if any were resolved by discussion. Similar codes were combined into themes [12]. To ensure that the results are a reflection of the data, the codes/themes were related back to the original data [16]. While representing the quotes, square brackets within quotes were used to mention authors' comments and round brackets were used to mention the sex and occupation of the participant. The findings were reported by using 'Consolidated Criteria for Reporting Qualitative Research [17].

\section{Results}

\section{Compliance to implementation of AIC guidelines}

The findings of non-participant observation of the six DR-TB centers are summarised in Table 1. In DR-TB wards $(n=6)$, compliance to hand hygiene, wet mopping and ventilation measures were satisfactory in four or more wards. All other components were found unsatisfactory in five or more wards. Visitor restriction and adherence to the use of personal protective equipment were not implemented in any of the wards.

All the patient waiting areas were adequately ventilated; however, patient fast tracking and cough hygiene components were unsatisfactory across the DR-TB centers. In the out-patient departments across the DR-TB centers, hand hygiene and ventilation components were satisfactory; however, the use of personal protective equipment for the health care providers was not implemented.

All the three CDST laboratories were consistently implementing all the AIC guidelines. Some exceptions were seen in the implementation of standard operating procedures, timely availability of lab reports and biosafety checklist in one laboratory.

About three fourths of the patients admitted in the DR-TB wards had a satisfactory knowledge of cough hygiene. Half and one third of the patients knew about the appropriate method for disposal of sputum and situations demanding mask usage respectively (Table 2).

KIIs confirmed the satisfactory implementation of wet mopping and adherence to hand hygiene. Two DR-TB wards had AIC compliant buildings and in the remaining four wards, existing hospital wards were structurally modified for in-patient treatment of DR-TB patients. We found that every patient was provided with a surgical mask on admission and educated about the seriousness of the illness, its transmission risk to others and cough hygiene.

The gaps in the effective implementation of AIC guidelines across administrative, environmental and personal protective categories have been summarised in Table 3. There were seven codes identified under these categories: lack of AIC compliant buildings, lack of visitor restriction, poor hand hygiene, unsafe sputum disposal, lack of training on AIC guidelines among staff, poor cross ventilation and lack of N95 mask.

\section{Reasons for unsatisfactory compliance}

The reasons for unsatisfactory compliance to AIC guidelines along with relevant quotes across health system and individual levels are summarized in Table 4. We identified four codes: poor coordination between RNTCP and general health services leading to lack of ownership; ineffective or non-existent infection control committees; vacant posts; and attitudes of health care delivery staff.

\section{Discussion}

This study is one of the few studies conducted in India to determine the implementation status of national AIC guidelines at DR-TB centres. The level of compliance with AIC guidelines among the DR-TB centers was sub-optimal when compared to the laid down guidelines (except for the CDST laboratories) at DR-TB wards, OPDs and patient waiting areas. As beneficiaries of the public health programme, there was scope for improving the existing patient knowledge with regard to cough hygiene. The findings related to compliance by non-participant observation were corroborated by KIIs. The recurring theme that emerged was a lack of investment and ownership as well as poor coordination between the national programme (RNTCP) and general health care system in the state as the main reason for unsatisfactory compliance.

\section{Strengths and limitations}

Operational research is essential to develop evidence-based policies to improve TB IC [9]. This is the first comprehensive study on the state-wide assessment of the AIC measures in the DR-TB centers in India. This assessment is not routinely done within the programmatic context; therefore it can serve as a baseline study for future research. There was a convergent validity between the findings of the quantitative and qualitative components. This makes our findings robust.

There were some limitations of the study. First, we were not able to get an insight into the pragmatic solutions to address the reasons for unsatisfactory compliance. The 
Table 1 Compliance to Airborne Infection Control guidelines among the DR-TB Centers in Karnataka, India, 2016-17 ( $n=6$ )

\begin{tabular}{|c|c|c|c|c|}
\hline SI. No & Facility assessed & AIC guidelines & Components of assessment & $\begin{array}{l}\text { Number of centers with } \\
\text { satisfactory compliance to } \\
\text { AIC guidelines }(n=6)\end{array}$ \\
\hline \multirow[t]{8}{*}{1} & \multirow[t]{8}{*}{ DR-TB Ward } & \multirow[t]{6}{*}{ Administrative } & Location and design & 1 \\
\hline & & & Visitor restriction & 0 \\
\hline & & & Hand hygiene & 4 \\
\hline & & & Cough hygiene & 1 \\
\hline & & & Wet mopping & 4 \\
\hline & & & Human resources training & 1 \\
\hline & & Environmental & Ventilation & 5 \\
\hline & & Personal Protective & Personal Protective Equipment & 0 \\
\hline \multirow[t]{4}{*}{2} & \multirow[t]{4}{*}{ Patient waiting area } & \multirow[t]{3}{*}{ Administrative } & Cough hygiene & 0 \\
\hline & & & Patient fastracking & 1 \\
\hline & & & Wet mopping & 4 \\
\hline & & Environmental & Ventilation & 6 \\
\hline \multirow[t]{5}{*}{3} & \multirow{5}{*}{$\begin{array}{l}\text { Chest medicine/ internal medicine } \\
\text { Out Patient Department }\end{array}$} & \multirow[t]{3}{*}{ Administrative } & Hand hygiene & 6 \\
\hline & & & Wet mopping & 4 \\
\hline & & & Human resources training & 3 \\
\hline & & Environmental & Ventilation & 5 \\
\hline & & Personal Protective & Personal Protective Equipment & 0 \\
\hline \multirow[t]{10}{*}{4} & \multirow[t]{10}{*}{ CDST Laboratory $(\mathrm{n}=3)$} & \multirow[t]{9}{*}{ Administrative } & Standard Operating Procedures & 2 \\
\hline & & & Human resources training & 3 \\
\hline & & & Signage & 2 \\
\hline & & & Lab reports & 2 \\
\hline & & & Bio-safety checklist & 2 \\
\hline & & & Hand hygiene & 3 \\
\hline & & & Location and design & 3 \\
\hline & & & Restricted entry & 3 \\
\hline & & & Sterilization & 3 \\
\hline & & Personal Protective & Personal Protective Equipment & 3 \\
\hline
\end{tabular}

$A / C$ airborne infection control, DR-TB drug resistant tuberculosis, CDST culture and Drug Sensitivity testing

staffs were not forthcoming with solutions. It could be because the nursing and housekeeping staffs were not informed a priori about the purpose of the visit. Second, an objective assessment of ventilation using appropriate tools for air flow and air velocity was not carried as we did not have the required scientific and allied resources.

Table 2 Knowledge about Cough Hygiene and Sputum Disposal of the patients admitted in DR-TB wards of DR-TB centers, Karnataka, India, 2016-17 ( $n=30)$

\begin{tabular}{lll}
\hline SI. No & Knowledge about the variables & $\begin{array}{l}\text { Satisfactory knowledge } \\
\mathrm{n}(\%)\end{array}$ \\
\hline 1 & Cough hygiene & $23(76.7)$ \\
2 & Purpose of use of surgical masks & $21(70.0)$ \\
3 & Situations that demand mask usage & $09(30.0)$ \\
4 & Disposal of sputum & $16(53.3)$ \\
\hline
\end{tabular}

\section{Interpretation of key findings}

A key finding in this study is that discrepancies between guidelines, standards and programme implementation with respect to operating processes and procedures can be traced to ambiguities at higher levels owing to a shortterm approach to institutional funding and design. These ambiguities percolate down through budgeting, hiring and contracting procedures. These asymmetries are palpable at multiple levels. At the scientific level, the variation of attitudes among doctors and nursing staff is one such asymmetry. At the level of human resource and staffing, short-term contracts compounded by low pay and lacking incentives to retain staff is another asymmetry. At the level of sensitizing staff, the lack of orientation sessions and on-going trainings is another. Finally, at the level of basic consumables, the lack of translating guidelines into budget-supported resources is yet another. 
Table $\mathbf{3}$ Gaps in the implementation of airborne infection control guidelines as perceived by the health care providers at DR-TB centers, Karnataka, India, 2016-17

\begin{tabular}{ll}
\hline Categories & Codes/ Theme \\
\hline Administrative & Lack of AIC \\
AlC measures & $\begin{array}{l}\text { guidelines comp } \\
\text { buildings }\end{array}$ \\
& \\
& Lack of visitor
\end{tabular}
restriction

Poor Hand Hygiene disposal

Lack of training on AIC guidelines among staff

Environmental Poor cross AIC measures ventilation Verbatim quotes

"There are patients with other illnesses in the same building just separated by few meters and a wall" (male doctor) "The wards are located too closely with the wards housing patients from other departments" (male doctor)

"Usually there will be one attender or sometimes two staying with the patient. Even when we tell about the disease and how it spreads, they tell that it is difficult to stay away." (female nurse) "As per our hospital policy, all the patients need to have one attender compulsorily with them at all the times." (female nurse)

\section{"When it comes to washing} there are no soaps provided for hand washing. Sinks are put up but no water is available. Running water is required; however, it is not there." (male nurse)

"Actually, each patient has to spit the sputum in the sputum cup prefilled with some water. The contents are poured in to the toilet and flushed." (female nurse) "There is presently no adequate supply of disinfectants; it may be phenol or even bleaching powder." (female housekeeping staff) "The patients are told to spit the sputum in the washroom and flush it with adequate water."

"I have not undergone any training. I have joined here just one year back." (female housekeeping staff) "I have not received any sort of training before getting posted here. Nobody who is posted here receives any sort of training." (female nurse) "There is attrition among the housekeeping staff and hence, a lot of new people are added to the pool regularly. Educating these people is also a challenge for us." (male doctor) "Due to administrative policy of the hospital, they [nurses] are relocated to different wards once in 2 months." (male doctor)

"Many a times at least during the nights, the windows are closed citing mosquito menace." (male doctor) (female housekeeping staff)
Table 3 Gaps in the implementation of airborne infection control guidelines as perceived by the health care providers at DR-TB centers, Karnataka, India, 2016-17 (Continued)

\begin{tabular}{|c|c|c|}
\hline Categories & Codes/ Themes & Verbatim quotes \\
\hline $\begin{array}{l}\text { Personal } \\
\text { Protective AIC } \\
\text { measures }\end{array}$ & Lack of N95 masks & $\begin{array}{l}\text { "We are trying our best to } \\
\text { provide the N95 masks. } \\
\text { However, we don't have supply } \\
\text { at all times." (male doctor) } \\
\text { "I don't feel very safe working in } \\
\text { this ward and always have the } \\
\text { fear of contracting the infection } \\
\text { (because of lack N95 mask)." } \\
\text { [female nurse] } \\
\text { "I use mask when I am working } \\
\text { in this ward and also in other } \\
\text { departments of the hospital. } \\
\text { This cloth mask!" (female nurse) } \\
\text { "I am not using N95 mask as } \\
\text { there is no supply. But what to } \\
\text { do? They are costly (to be } \\
\text { purchased)." (male doctor) }\end{array}$ \\
\hline
\end{tabular}

$D R-T B$ drug resistant tuberculosis

The N95 mask is one of the most important preventive measures for frontline health workers working in a high risk setting and is expected to reduce or even break the transmission cycle $[10,18]$. Unfortunately, the nonavailability of N95 masks in wards as well as OPDs was staggering. They were not available as a rule and if available, they were not provided to the nursing or housekeeping staff. The consistent non-availability of N95 masks was also pointed out in another study from South Africa [19].

On the side of patients, they were encouraged to dispose sputum either in the sink or the toilet without prior disinfection, posing a risk for transmission of MDR-TB, as observed in our study. At a pan-centre level, the lack of display materials on cough hygiene and patient education were not found in the wards and OPD waiting areas in most of the centers. These display boards act as reinforcement for health promoting behaviour and are a low-cost, highimpact way of educating multiple stakeholders [10].

Patient fast tracking in OPD waiting areas and visitor restrictions are other obvious measures which minimise the exposure of susceptible people to MDR-TB. These practices were not implemented in our setting. This is a major finding. It almost destroys the purpose of having a separate DR-TB ward. Finally, no policy or guideline can be successful without inviting the intelligent investments of key implementing stakeholders: the participants in our study reported that they have not undergone any training on AIC measures. Considering the frequent shifting of the nurses (which is a policy of the hospitals) and attrition among housekeeping staff; this is a vital point that needs to be addressed. Similar findings were reported in other studies from developing countries [11, 19, 20].

These gaps in AIC were contrary to one of the studies from South Africa where all the facilities assessed were found to have displayed signage to educate patients and 
Table 4 Provider perspectives into barriers for implementation of airborne infection control guidelines at DR-TB centers, Karnataka, India, 2016-17

\begin{tabular}{lll}
\hline Categories & Codes/Themes & Verbatim quotes \\
\hline Health & Poor coordination leading & "The programme gives us a
\end{tabular}

system to lack of ownership one-time grant to set up the level ward and other required modifications; however there is no supply of consumables [ex. masks and disinfectants] by the programme at any point of time." (male doctor) "We are not directly involved in the implementation of the TB prevention programme. We supplement it as a part of the teaching institution attached to that." (male doctor)

"N95 mask has to be supplied to all the medical and para medical staff by the hospital or program. There should be some display material provided by the program regarding cough hygiene and nutrition." (male doctor)

"We have placed an indent for these items required many a times but they are not supplied. We have even brought it to the notice of the district programme officials; however, it was told that it is the responsibility of the hospital to provide these." (male doctor) "As per my rough estimate, we require about 300 odd N95 masks a month if it has to be used properly among all cadres of staff. Who has to supply this when each costs atleast a dollar per piece? There is always a tussle going on between the programme officials and the hospital authorities regarding the resources. The problem lies in "who has to do it at the end of the day?" (male doctor)

Ineffective or non-existent "We don't have any regular Infection control (IC) meetings of the IC Committee committees

Vacant posts
Table 4 Provider perspectives into barriers for implementation of airborne infection control guidelines at DR-TB centers, Karnataka, India, 2016-17 (Continued)

\begin{tabular}{|c|c|c|}
\hline Categories & Codes/ Themes & Verbatim quotes \\
\hline & & $\begin{array}{l}\text { nurses against the sanctioned } \\
\text { number." (male doctor) } \\
\text { "Medical officers get trained } \\
\text { and they leave after working } \\
\text { here for some time and getting } \\
\text { an experience." (male doctor) } \\
\text { "Medical officers are recruited } \\
\text { on contractual basis and are } \\
\text { poorly paid. There is no risk } \\
\text { allowance for working in DR TB } \\
\text { center." (male doctor) }\end{array}$ \\
\hline $\begin{array}{l}\text { Individual } \\
\text { level }\end{array}$ & $\begin{array}{l}\text { Attitudes of health care } \\
\text { delivery staff }\end{array}$ & $\begin{array}{l}\text { "I don't think exhaust fans are } \\
\text { useful. The number of patients } \\
\text { are very much less here, may } \\
\text { be around 8-10 patients at the } \\
\text { maximum. In that case for in- } \\
\text { fection control, we don't need } \\
\text { these things." (male doctor) } \\
\text { "N95 masks are available only } \\
\text { for the doctors; cloth masks are } \\
\text { made available for the nurses } \\
\text { and housekeeping staff." (male } \\
\text { doctor) } \\
\text { "I don't feel any risk to the } \\
\text { nursing staff. None amongst us } \\
\text { have suffered from any type of } \\
\text { Tuberculosis." (male nurse) }\end{array}$ \\
\hline
\end{tabular}

$D R$-TB drug resistant tuberculosis

health care workers on cough hygiene. N95 mask was uniformly available for use among the health care workers but they were not used. However, the ward visits by the visitors were a common practice similar to that in our study [21].

There were two main reasons for unsatisfactory compliance. First, there was poor coordination between RNTCP and general health care system (hospital authorities) leading to lack of ownership among the administrators, nodal officers and medical officers. After the one time grant by RNTCP to implement AIC guidelines in DR-TB centers, roles and responsibilities have been clearly demarcated across human resources. Though, it is clear from the national guidelines that the hospital authorities have to run the DR-TB center [3], there was lack of clarity regarding the onus of responsibility for financial resources, for acquiring the consumables such as N95 masks and phenol for sputum disinfection among others. The hospital authorities/ doctors did not consider it as their primary responsibility resulting in gaps in AIC. Vacant posts of medical officer (in four out of six DR-TB centers) could further contribute to this lack of ownership and also might be a deterrent for the on job training of the nursing and housekeeping staff in AIC measures. This is particularly important as the nodal officer is a specialist who also holds additional clinical and faculty positions and may not have the time 
for day to day on the job training and supervision of nursing and housekeeping staff.

Second, the IC committees were either non-existent or non-functional. Similar findings were observed in studies from India, South Africa and Pakistan [11, 19, 20]. However, another South African study reported that about $80 \%$ of the DR-TB centers assessed had IC committees [21]. Hospital IC committees are expected to perform risk assessment, prepare facility infection control plan incorporating the AIC component as well. IC committees need to have a realistic budget for carrying out and ensuring implementation of IC [10]. These activities including training of health staff were probably not being carried out resulting in gaps in AIC implementation. The use of simple videos in the local language can easily be rolled out to sensitize staff to how preventive measures are within reach. However, this is the responsibility of IC committees.

Based on the codes generated in our analysis (Tables 3 and 4) and the above interpretations, a visual framework of the reasons leading to gaps in implementation (unsatisfactory compliance) of AIC guidelines is depicted in Fig. 2.

\section{Recommendations for policy and practice}

Based on our study findings, the following recommendations can be drawn. First, the administrators of the hospitals need to reinvigorate the existing infection control committees so that they perform their duties as per the suggested guidelines [10]. The programme needs to routinely incorporate the review of IC meetings held and AIC measures undertaken at DR-TB centers during the quarterly and national review meetings. Second, hospital authorities need to take the ownership of AIC in DR-TB centers and make budgetary allocations towards display of patient education on cough hygiene in prominent locations in the local languages and consumables like N95 masks, sputum cups and phenol for sputum disinfection. Third, standard operating procedures related to AIC for doctors, nurses as well as housekeeping staff should be displayed near their workstations in the local language. Fourth, vacant posts of Medical officers need to be filled up at the earliest by RNCTP and financial mechanisms need to be evolved to increase retention of the medical officers. Further research in deployment of newer technologies and equipments to measure and maintain airflow in the wards has to be prioritized.

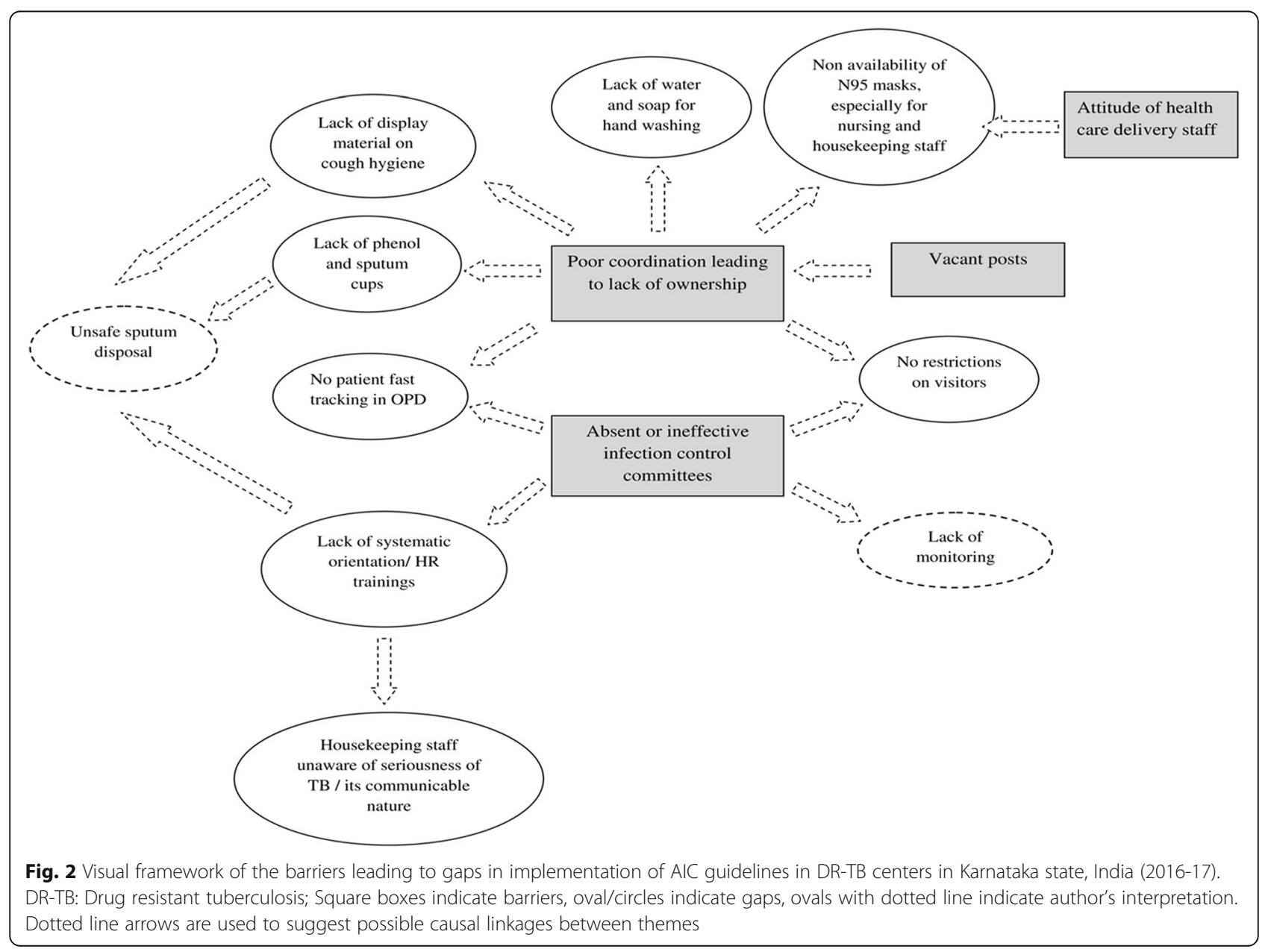




\section{Conclusions}

Compliance with AIC guidelines in the DR-TB centers of the state of Karnataka, India has a scope for improvement. The reasons for unsatisfactory compliance require urgent attention of the programme managers and hospital authorities, especially the latter, as the DR-TB centers are administratively under them. The gravity of MDR-TB (and the low cure rate of around 54\% with the existing drug regimens) underlines the significance of taking long-sighted measures for a potentially dangerous disease that can be controlled with some investment of capital, administrative and scientific resources [22].

\section{Additional file}

Additional file 1: Annex S1. Operational definitions used for assessing compliance of DR-TB centers towards AIC measures in Karnataka, India, 2016-17. Annex S2. Operational definitions used to assess Knowledge about Cough Hygiene and Sputum Disposal of the patients admitted in DR-TB wards of DR-TB centers, Karnataka, India, 2016-17. Annex S3. Interview guides used for key informant interviews at Drug Resistant Tuberculosis (DR-TB) centres of Karnataka, India, 2016-17. (DOCX 25 kb)

\section{Abbreviations}

AIC: Airborne infection control; CDST: Culture and Drug Sensitivity testing; DR-TB: Drug resistant tuberculosis; IC: infection control; KIl: Key informant interview; MDR-TB: Multidrug resistant tuberculosis; OPD: outpatient department; PMDT: programmatic management of drug resistant TB; RNTCP: Revised national tuberculosis control programme

\section{Acknowledgements}

This research was conducted through the Structured Operational Research and Training Initiative (SORT IT), a global partnership led by the Special Programme for Research and Training in Tropical Diseases at the World Health Organization (WHO/TDR). The model is based on a course developed jointly by the International Union Against Tuberculosis and Lung Disease (The Union) and Medécins sans Frontières (MSF/Doctors Without Borders). The specific SORT IT programme which resulted in this publication was jointly developed and implemented by: The Union South-East Asia Office, New Delhi, India; the Centre for Operational Research, The Union, Paris, France; the Operational Research Unit (LUXOR), MSF Brussels Operational Center, Luxembourg; Department of Preventive and Social Medicine, Jawaharlal Institute of Postgraduate Medical Education and Research, Puducherry, India; Department of Community Medicine, Pondichern Institute of Medical Sciences, Puducherry, India; Department of Community Medicine, Sri Manakula Vinayagar Medical College and Hospital, Puducherry, India; Department of Community Medicine, Velammal Medical College Hospital and Research Institute, Madurai, Tamil Nadu; Narotam Sekhsaria Foundation, Mumbai, India; and National Institute for Research in Tuberculosis, Chennai, India. We would like to thank the study participants for their valuable time and insights. We would like to thank Dr. Manjunath S Nekar, Assistant Professor, Department of Community Medicine, Karnataka Institute of Medical Sciences, Hubballi and Dr. Gururaj N A, Assistant Professor, Gulburga Institute of Medical Sciences, Kalaburgi for their help in local coordination. We also acknowledge the administrators of the hospitals which had DR-TB centers for their approvals to conduct the study. We place on record the support provided by the State TB office, Karnataka and the staff of RNTCP working at the study sites for their help in coordinating with the hospital authorities.

\section{Funding}

The training programme and open access publications costs were funded by the Department for International Development (DFID), UK and La Fondation Veuve Emile Metz-Tesch (Luxembourg). The funders had no role in study design, data collection and analysis, decision to publish, or preparation of the manuscript.

\section{Availability of data and materials}

Data and de-identified interview transcripts are available on request from the corresponding author.

\section{Authors' contributions}

Principal Investigator and corresponding author: KMA; mentors: HDS, AG, OPA; senior author: AGJ; conception / design of the study: KMA, HDS, OPA, AGJ, SBN; data collection, data entry and transcription: KMA, SBN, ASN; data analysis and interpretation: KMA, OPA, HDS, OPA, AGJ; preparing the first draft: KMA, HDS, AS; critical comments and approval of the final draft: all authors. All authors read and approved the final manuscript.

\section{Ethics approval and consent to participate}

Ethics approval was obtained from the Institutional Ethics Committee of Yenepoya University, Mangaluru, India (protocol number YUEC/2016/288, approval dated 12/11/2016) and the Ethics Advisory Group of the International Union against Tuberculosis and Lung Disease (The Union), Paris, France (EAG No. 92/16 dated 17/10/2016). Written informed consent was taken from the relevant authorities for facility observation, the same was obtained from patients for structured interview and from health care providers for key informant interviews. Permission was taken from the Joint Director, Tuberculosis of the state of Karnataka before initiating the study We decided against providing age of participant whose quotes were used to protect the identity of the participant. The investigator used N95 mask while interviewing the patients and assessing the AIC compliance in DR-TB centers,

Consent for publication

Not applicable.

\section{Competing interests}

The authors declare that they have no competing interests.

\section{Publisher's Note}

Springer Nature remains neutral with regard to jurisdictional claims in published maps and institutional affiliations.

\section{Author details}

${ }^{1}$ Department of Community Medicine, Yenepoya Medical College, Yenepoya University, Mangaluru 575018, India. ${ }^{2}$ International Union against Tuberculosis and Lung Diseases, South East Asia Office, New Delhi, India. ${ }^{3}$ Department of Community Medicine, Government Medical College, Thrissur, India. ${ }^{4}$ Department of Community Medicine, ESIC Medical College and PGIMSR, Bengaluru, India. ${ }^{5}$ Department of Health and Family Welfare, Government of Karnataka, Bengaluru, India.

Received: 18 July 2017 Accepted: 26 October 2017 Published online: 06 November 2017

\section{References}

1. World Health Organization. Global TB Report 2016. Geneva; 2016. Available at http://apps.who.int/iris/bitstream/10665/250441/1/9789241565394-eng. pdf?ua=1. Accessed 14 June 2017.

2. Central TB Division Directorate General of Health Services Ministry of Health and Family Welfare. TB India 2017. New Delhi; 2017. Available at https:// tbcindia.gov.in/WriteReadData/TB\%20India\%202017.pdf. Accessed 14 June 2017.

3. Central TB Division Directorate General of Health Services Ministry of Health and Family Welfare. Guidelines on Programmatic Management of Drug Resistant TB (PMDT) in India. New Delhi; 2012. Available at https://tbcindia. gov.in/WriteReadData//892s/ 8320929355Guidelines\%20for\%20PMDT\%20in\%20India\%20\%20May\%202012.pdf. Accessed 13 Sept 2016.

4. Jarand J, Shean K, O'Donnell M, Loveday M, Kvasnovsky C, Van der Walt M, et al. Extensively drug-resistant tuberculosis (XDR-TB) among health care workers in South Africa. Trop. Med. Int Heal. 2010;15:1179-84.

5. Gandhi NR, Weissman D, Moodley P, Ramathal M, Elson I, Kreiswirth BN, et al. Nosocomial transmission of extensively drug-resistant tuberculosis in a rural hospital in South Africa. J Infect Dis. 2013;207:9-17.

6. Williams OM, Abeel T, Casali N, Cohen K, Pym AS, Mungall SB, et al. Fatal Nosocomial MDR TB identified through routine genetic analysis and wholegenome sequencing. Emerg Infect Dis. 2015;21:1082-4. 
7. Bantubani N, Kabera G, Connolly C, Rustomjee R, Reddy T, Cohen T, et al. High rates of potentially infectious tuberculosis and multidrug-resistant tuberculosis (MDR-TB) among hospital inpatients in KwaZulu Natal, South Africa indicate risk of nosocomial transmission. Pai M, editor. PLoS One 2014;9:e90868

8. World Health Organization. WHO Policy on TB Infection Control in HealthCare Facilities, Congregate Settings and Households. Geneva; 2009. Available at http://apps.who.int/iris/bitstream/10665/44148/1/ 9789241598323_eng.pdf. Accessed 13 Sept 2016.

9. The Tuberculosis Coalition for Technical Asssistance (TBCTA). Implementing the WHO Policy on TB Infection Control in Health-Care Facilities, Congregate Settings and Households. Atlanta; 2009. Available at http:// www.stoptb.org/wg/tb_hiv/assets/documents/ TBICImplementationFramework1288971813.pdf. Accessed 14 June 2017.

10. Directorate General of Health Services Ministry of Health and Family Welfare Government of India. Guidelines on Airborne Infection Control in Healthcare and Other Settings; 2010. Available athttp:/lupsacs.in/pdf/ Guidelines_on_Airborne_Infection_Control_April2010Provisional.pdf. Accessed 13 Sept 2016.

11. Parmar MM, Sachdeva KS, Rade K, Ghedia M, Bansal A, Nagaraja SB, et al. Airborne infection control in India: baseline assessment of health facilities. Indian J Tuberc. 2015;62:211-7.

12. Creswell J, Plano CV. Designing and conducting mixed methods research. London (United Kingdom): Sage Publications Ltd; 2007.

13. Government of India Ministry of Home Affairs Office of Registrar General and Census Commissioner; 2011. Available athttp://www.censusindia.gov.in/ 2011-prov-results/data_files/karnataka/Size_growth_population_39_62.pdf. Accessed 14 June 2017.

14. Kvale S. Doing interviews. London: SAGE Publications; 2007.

15. Saldana J. The coding manual for qualitative research. Los Angeles: SAGE Publications; 2010

16. Lincoln YS, Guba EG. Naturalistic inquiry. New York: SAGE Publications, Inc; 1985.

17. Tong A, Sainsbury P, Craig J. Consolidated criteria for reporting qualitative research (COREQ): a 32-item checklist for interviews and focus groups. Int J Qual Health Care. 2007:19:349-57.

18. Basu S, Andrews J, Poolman EM, Gandhi NR, Shah NS, Moll AP, et al. The epidemic level impact of preventing nosocomial transmission of XDR TB in rural south african district hospital. Lancet. 2007;370:1500-7.

19. Zelnick JR, Gibbs A, Loveday M, Padayatchi N, O'Donnell MR. Health-care workers? Perspectives on workplace safety, infection control, and drugresistant tuberculosis in a high-burden HIV setting. J Public Health Policy. 2013;34:388-402.

20. Waheed Y, Khan MA, Fatima R, Yaqoob A, Mirza A, Qadeer E, et al. Infection control in hospitals managing drug-resistant tuberculosis in Pakistan: how are we doing? Public heal. Action. 2017;7:26-31.

21. Farley JE, Tudor C, Mphahlele M, Franz K, Perrin NA, Dorman S, et al. A national infection control evaluation of drug-resistant tuberculosis hospitals in South Africa. Int J Tuberc Lung Dis. 2012;16:82-9.

22. Ahuja SD, Ashkin D, Avendano M, Banerjee R, Bauer M, Bayona JN, et al. Multidrug resistant pulmonary tuberculosis treatment regimens and patient outcomes: an individual patient data meta-analysis of 9,153 patients. Evans C, editor. PLoS med. Public Libr Sci. 2012;9:e1001300.

\section{Submit your next manuscript to BioMed Central and we will help you at every step:}

- We accept pre-submission inquiries

- Our selector tool helps you to find the most relevant journal

- We provide round the clock customer support

- Convenient online submission

- Thorough peer review

- Inclusion in PubMed and all major indexing services

- Maximum visibility for your research

Submit your manuscript at www.biomedcentral.com/submit
Biomed Central 